\title{
Genetic marking and characterization of Tac2- expressing neurons in the central and peripheral nervous system
}

\author{
Lynn Mar, Fu-Chia Yang and Qiufu Ma*
}

\begin{abstract}
Background: The neurocircuits that process somatic sensory information in the dorsal horn of the spinal cord are still poorly understood, with one reason being the lack of Cre lines for genetically marking or manipulating selective subpopulations of dorsal horn neurons. Here we describe Tac2-Cre mice that were generated to express the Cre recombinase gene from the Tac2 locus. Tachykinin 2 (Tac2) encodes a neurotransmitter, neurokinin B (NKB).

Results: By crossing Tac2-Cre mice with ROSA26-tdTomato reporter mice, we directly visualized Tac2 lineage neurons in the dorsal root ganglia, the dorsal horn of the spinal cord, and many parts of the brain including the olfactory bulb, cerebral cortex, amygdala, hippocampus, habenula, hypothalamus, and cerebellum. This Tac2-Cre allele itself was a null allele for the Tac2 gene. Behavioral analyses showed that Tac2 homozygous null mice responded normally to a series of algogenic (pain-inducing) and pruritic (itch-inducing) stimuli.

Conclusions: Tac2-Cre mice are a useful tool to mark specific subsets of neurons in the sensory ganglia, the dorsal spinal cord, and the brain. These mice can also be used for future genetic manipulations to study the functions of Tac2-expressing neurons or the functions of genes expressed in these neurons.
\end{abstract}

\section{Background}

The spinal cord dorsal horn has long been recognized as the processing center for sensory information including pain, itch, cold, and warmth [1-4]. The dorsal horn is divided into five laminae, which can be identified by lamina-specific innervation by primary sensory fibers and by the expression of molecular markers [3]. For example, neurons in superficial laminae that express the neurokinin 1 receptor (NK1R) and the gastrin-releasing peptide receptor (GRPR) are involved in sensing pain and/or itch [5-7]. Neurons in the inner layer of lamina II that express protein kinase $\mathrm{C} \gamma(\mathrm{PKC} \gamma)$ are the targets of myelinated non-nociceptive afferents [8], and mediate nerve injury-induced mechanical allodynia, a type of pain evoked by innocuous mechanical stimuli [9]. Despite significant progress, the neural circuits that process specific somatic sensory information in the dorsal horn are still not well characterized [3].

\footnotetext{
* Correspondence: qiufu_ma@dfci.harvard.edu

Dana-Farber Cancer Institute and Department of Neurobiology, Harvard Medical School, 450 Brookline Ave, Boston, Massachusetts USA 02215
}

Tachykinin peptides are encoded by the tachykinin 1 (Tac1) and tachykinin 2 (Tac2) genes and are known to modulate neuronal activity [10]. Tac1 encodes a precursor protein that produces two peptides, substance P (SP) and neurokinin A (NKA), whereas Tac2 encodes neurokinin B (NKB). Release of SP from sensory neurons in the dorsal root ganglia (DRG) plays a crucial role in modulating pain and itch [11-13]. More specifically, mice lacking Tac1 fail to sense moderate to intense thermal and mechanical pain [11]. Tac2 expression has also been detected in restricted neuronal populations in the central nervous system [14-16]. In the dorsal spinal cord, for example, Tac2 was detected in lamina I and the inner layer of lamina II of both mouse and rat dorsal horns [14-16]. Consistently, it has been suggested that activation of NK3R, the NKB receptor, plays a role in pain modulation [17-19].

The Cre-loxP recombinase system has become a powerful tool to study the morphology and function of specific neuronal populations. An exemplary triumph is the creation of Cre mouse lines that mark distinct subsets of primary sensory neurons, which allowed precise
Ciomed Central

() 2012 Mar et al; licensee BioMed Central Ltd. This is an Open Access article distributed under the terms of the Creative Commons Attribution License (http://creativecommons.org/licenses/by/2.0), which permits unrestricted use, distribution, and reproduction in any medium, provided the original work is properly cited. 
genetic ablation of Cre-expressing sensory neurons, and thus have provided unprecedented insight into the cellular basis of pain and itch [20-23]. The availability of additional precise and neuron-specific Cre lines will allow more such discoveries, especially in light of genome-wide conditional knockout resources [24]. To study the physiological function of Tac2, and to characterize Tac2-expressing neurons, here we report the generation of Tac2-Cre mice in which Cre recombinase is under the control of the Tac2 locus, and where Tac2-Cre represents a null allele.

\section{Results}

\section{Generation of Tac2-Cre mice}

The targeting strategy used to generate Tac2-Cre mice is illustrated in Figure 1. In the targeting vector, Cre and a neomycin selection cassette were inserted into exon 3 , the first coding exon of Tac2. The neomycin selection cassette is floxed with FRT sites that can be removed later by Flipase-mediated DNA recombination [25]. After electroporation in $129 / \mathrm{Sv}$ embryonic stem cells, 192 clones that survived selection with Geneticin were screened via Southern blot analysis, and 9 correctly targeted clones were identified. The digestion of genomic DNA with the restriction enzyme XbaI generated $\mathrm{a} 12 \mathrm{~Kb}$ fragment for the wildtype allele, and two different $7 \mathrm{~Kb}$ fragments for the Tac2-Cre mutant allele, depending on whether the 5' or 3' arm external probe was used (Figure 1). Two positive cell lines were used for blastocyst

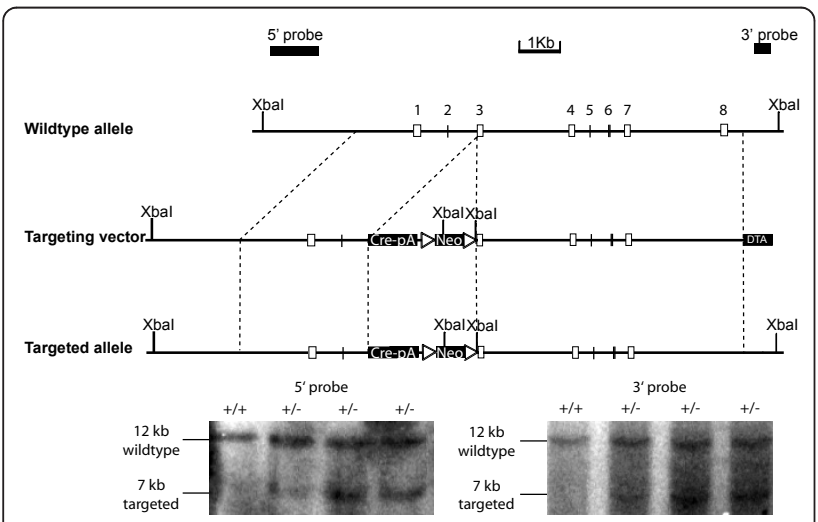

Figure 1 Generation of Tac2-Cre mice. Top, schematic diagram of the gene targeting strategy used to insert Cre into the wildtype Tac2 locus. The Tac2-Cre targeting vector included Cre directly downstream of the Tac2 ATG codon sequence, an FRT-flanked neomycin positive selection cassette, and a DTA negative selection cassette. Xbal sites and the expected sizes of the resulting DNA fragments are indicated. Below, Southern blot analyses for verification of correct targeting of Tac2 locus. Southern blot with Xbal and external probes confirmed correctly targeted homologous recombination events. The $12 \mathrm{~kb}$ WT band is seen in all lanes, and two correctly targeted and distinct $7 \mathrm{~Kb}$ bands represent the targeted allele at the $5^{\prime}$ and $3^{\prime}$ homology arms. injection and the resulting chimera mice were crossed with C57BL6 wildtype mice. F1 progenies carrying germ line transmission of the Tac2-Cre allele were identified, which were then crossed to FLPeR mice to delete the neomycin cassette [25].

\section{Tac2-Cre expression in the dorsal spinal cord}

To analyze the efficiency and fidelity of Cre recombinase activity, Tac2-Cre mice were crossed to the Cre-dependent reporter line ROSA26-loxP-STOP-loxP-tdTomato [26]. The resulting double heterozygous mice (Tac2-Cre; ROSA26-loxP-STOP-loxP-tdTomato) will be referred to as Tac2-Cre/Tomato mice, in which Cre-mediated recombination led to constitutive expression of the red fluorescent protein Tomato. As a result, neurons that expressed Tac2-Cre, either transiently or persistently, were permanently marked by Tomato expression.

In the dorsal spinal cord of Tac2-Cre/Tomato mice, Tomato expression was first detected at postnatal day 4, similar to in situ expression analysis ([27], data not shown). By P30, Tomato-positive neurons were observed mainly within the ventral layer of inner lamina II and lamina III (Figure 2A, big arrow; Figure 2B), as they were located ventral to primary sensory terminals labeled by isolectin-B4 (IB4) that innervate the dorsal layer of inner lamina II (Figure 2C) [8]. A few scattered Tomato-expressing cells were also observed in more ventral spinal areas (Figure 2A, small arrow). Furthermore, the processes of these Tomato-positive neurons were short and remained within the narrow laminae (Figure 2B, small arrow; Figure 2E, small arrow), suggesting their role in processing local information. Occasionally, Tomato-expressing neurons were also found in lamina I (Figure 2B, arrowhead), and they exhibited long, transverse processes (Figure 2D). This lamina-specific distribution of Tac2-Cre/Tomato neurons is consistent with the reported distribution of Tac2 mRNA and NKB peptide [14-16,27].

To further examine efficacy and specificity, we performed double staining of the Tomato protein and Tac2 mRNA, transcribed from the wildtype allele and detected by in situ hybridization, on spinal sections of P30 Tac2-Cre/Tomato heterozygous mice. We found Tomato reporter expression in the majority (169/209, $81 \%$ ) of neurons with detectable Tac2 transcripts (Figure 2E, arrow), demonstrating that Tac2-Cre was able to drive efficient DNA recombination in most Tac2-expressing neurons. However, we also found that a significant number of Tomato-expressing dorsal horn neurons failed to show detectable Tac2 mRNA expression (Figure 2E, arrowhead). Notably, the Tac2-negative, Tomato-positive neurons intermingled with Tac2-positive neurons in the same dorsal horn laminae (Figure 2E). Thus, some dorsal horn neurons expressed Tac2 





either transiently or at levels below detection using standard in situ hybridization techniques, although we cannot rule out the possibility that inserting Cre within the Tac2 coding sequence led to ectopic expression of Tac2-Cre.

Next, we conducted molecular analysis on these Tac2Cre/Tomato neurons. PKC $\gamma$ is expressed by neurons within ventral layer of inner lamina II, and 20\% (32/160) of Tomato-positive neurons of Tac2-Cre/Tomato mice clearly expressed PKC $\gamma$ (Figure 2F, arrow). The remaining Tomato-expressing neurons were located in areas that exhibited weak PKC $\gamma$ staining (Figure 2F, arrowhead). This weak PKC $\gamma$ staining may be indicative of weak PKC $\gamma$ expressing neurons, or represent axonal or dendritic processes derived from adjacent strong PKC $\gamma$ expressing neurons. If the latter is true, this subset of Tomato-positive neurons may not express $\mathrm{PKC} \gamma$ per se, but instead connect with PKC $\gamma$-expressing neurons.

We reported previously that Tac 2 mRNA in the dorsal horn is mainly detected in glutamatergic excitatory neurons marked by the expression of Tlx3, a homeobox protein [27]. Consistent with this, most Tac2-Cre/ Tomato-positive neurons $(85 \%, 167 / 197)$ continued to express Tlx3 (Figure 2G, arrow) [27,28], and almost none of the Tac2-Cre/Tomato-positive neurons coexpressed Pax2 (2\%, 2/131), a marker of inhibitory neurons (Figure $2 \mathrm{H}$, green) [28].

\section{Tac2-Cre/Tomato expression in the brain}

Tomato-positive neurons were detected in multiple brain regions as shown in a sagittal brain section of a P30 Tac2-Cre/Tomato mouse (Figure 3), and this result is largely consistent with previous expression analyses $[14,15]$. Within the main olfactory bulb, Tomato labeled neurons were located within the periglomerular layer (Figure 3A, B) and the granule layer (Figure 3A, C). Notably, labeled neurons in the granule layer sent long apical dendrites (Figure 3C), a morphology typical of inhibitory granule cells as reviewed by Shepherd et al. [29]. In the cerebral cortex, Tomato fluorescence was enriched in layers II and III, but was also found scattered through other cortical layers (Figure 3A, D). These neurons showed pyramidal shaped soma with long dendrites toward the apical surface (Figure 3D), a morphology typical of glutamatergic projection neurons [30]. In the hippocampus, Tomato was expressed in the granule cell layer of the dentate gyrus and the pyramidal cell layer of the CA1-CA3 regions (Figure 3A, E, Figure 4A). Other Tomato-expressing neurons were observed in the bed nuclei of the stria terminalis (Figure 3A, G), the granule cells in the cerebellum (Figure $3 \mathrm{~A}, \mathrm{H}$ ), and the superficial laminae of the spinal trigeminal nuclei (Figure 3A, I). Furthermore, a coronal section demonstrated Tomato expression in the medial habenula (Figure 4A,
B), amygdala nuclei (Figure 4A, C), and scattered neurons in hypothalamic nuclei including the arcuate nucleus (Figure 4A, D). In contrast, Tomato expression was rarely detected in other brain regions such as the thalamus (Figure 3A, F).

\section{Tac2-Cre/Tomato expression in the peripheral nervous system}

Within the peripheral nervous system, Tac2-Cre/Tomato was detected in a small subset of neurons in the DRG (Figure 5A) and in the sympathetic ganglia (data not shown). This is surprising because detection of Tac2 mRNA in adult DRG has not yet been reported ([10,14], data not shown). Given Tomato reporter expression was present at P0 (data not shown), these neurons might express Tac 2 transiently during embryonic development. Interestingly, these Tomato-positive neurons innervated selective peripheral targets (Figure 5B-D). Wholemount preparations revealed Tomato-positive fibers in the glabrous skin (Figure $5 \mathrm{~B}$ ), and as shown in a transverse section, these fibers were located within the dermal layer (Figure 5C). In the colon, a visceral organ, thin circumferential Tomato-positive fibers were detected in the muscle layer (Figure 5D), and Tac2-Cre expression was also detected in the bladder (data not shown).

\section{Tac2 null mice responded normally to pain-related or itch-related stimuli}

In Tac2-Cre mice, the Cre expression cassette, which contained the HSV thymidine kinase polyA signal, was inserted directly after the first ATG codon of the Tac2 gene, thereby creating a null allele. Consistent with this, we demonstrated a loss of Tac2 expression in the dorsal spinal cord of mutant mice (Figure 6A). In contrast, a control in situ hybridization using the somatostatin probe showed comparable signals in mutant versus control littermates (Figure 6A). Tachykinin peptides, such as SP and NKA, have been implicated in modulating pain and itch [11-13,31]. Therefore we sought to determine if Tac2 also played a role in modulating pain and/ or itch sensation. Tac $2^{\mathrm{Cre} / \mathrm{Cre}}$ homozygous null mice survived at the expected Mendelian ratio. We performed a series of pain and itch behavior assays on these mutant mice, using wildtype or $\mathrm{Tac} 2^{\mathrm{Cre} /+}$ heterozygous littermates as controls. In comparison to control mice, Tac $2^{\text {Cre/Cre }}$ homozygous null mice responded normally to painful stimuli, including noxious heat $\left(48^{\circ} \mathrm{C}, 50^{\circ} \mathrm{C}\right.$, $52^{\circ} \mathrm{C}, 54^{\circ} \mathrm{C}, 56^{\circ} \mathrm{C}, 58^{\circ} \mathrm{C}$, Figure $\left.6 \mathrm{~B}\right)$, light mechanical stimuli (von Frey assay, Figure 6C), intense mechanical stimuli (Randall-Selitto; Figure 6D), and chemical stimuli (formalin, Figure 6E). Moreover, like control littermates, Tac $2^{\text {Cre/Cre }}$ homozygous null mice showed similar scratching responses following nape injection of itching compounds [23,32,33], including Compound 48/80 

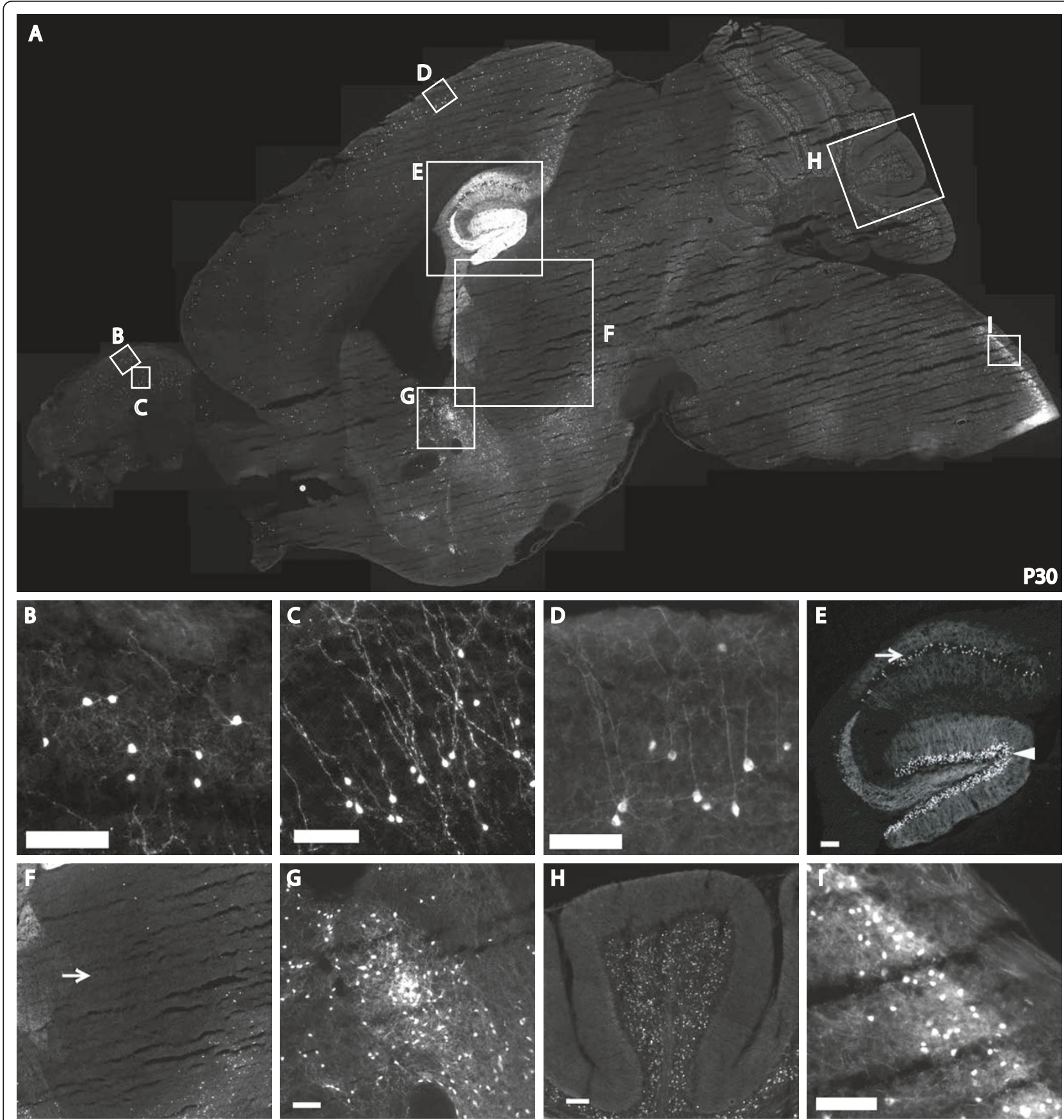

Figure 3 Tac2-Cre-expressing neurons in multiple brain regions. A sagittal section of a P30 adult Tac2-Cre/ROSA26-tdTomato brain (A) showing Tomato positive neurons were located within the olfactory bulb $(\mathbf{B}, \mathbf{C})$, cortex $(\mathbf{D})$, hippocampus (E), bed nuclei of the stria terminalis $(\mathbf{G})$, cerebellum $(\mathbf{H})$, medulla $(\mathbf{I})$, but not in all the brain regions, such as the thalamus $(\mathbf{F}$, arrow). Arrowhead and arrow in $\mathbf{E}$ indicate the dentate gyrus and the CA1-CA3 regions of the hippocampus, respectively. Scale bar, 50 um.

(Figure 6F), PAR2 agonist (Figure 6G), chloroquine (Figure $6 \mathrm{H}$ ), and serotonin (Figure 6I).

\section{Discussion}

We have generated a Tac2-Cre knock-in mouse line that drives Cre recombinase expression in various parts of the nervous system, including sensory neurons innervating skin dermis and visceral organs, a subset of excitatory neurons in the dorsal spinal cord, interneurons in the olfactory bulb, projection neurons in the cerebral cortex, neurons in specific amygdala nuclei, granule cells in the dentate gyrus, pyramidal neurons in the 


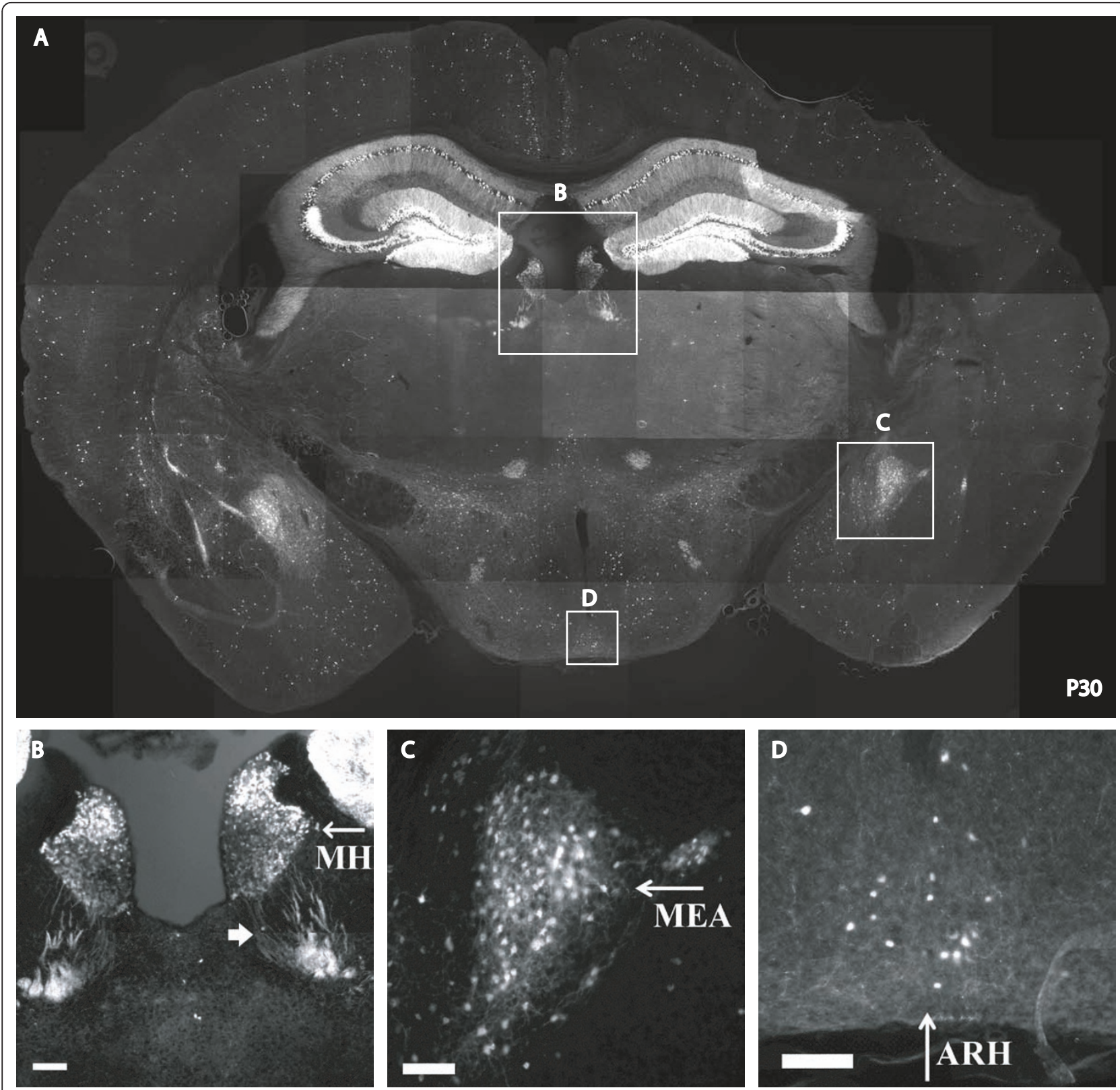

Figure 4 Tac2-Cre neurons located in additional brain regions. A transverse section of a P30 adult Tac2-Cre/ROSA26-tdTomato brain showing Tomato-positive neurons $(\mathbf{A})$. Note Tomato expression within the medial habenula $(\mathbf{B}$, "MH"), with processes projecting ventral laterally $(\mathbf{B}$, arrowhead). Tomato expression was also detected in the medial amygdalar nucleus (C, "MEA"), and the arcuate hypothalamic nucleus (D, "ARH"). Scale bar, 50 um.

hippocampal CA1-CA3 regions, granule cells in the cerebellum, and others. Thus, this Cre line will be of broad utility to the neuroscience community.

In the dorsal spinal cord, Tac2-Cre is expressed in the ventral layer of inner lamina II and lamina III, and occasionally in lamina $I$ and in more ventral spinal areas (Figure 2A, B). Tac2-Cre neurons in the dorsal horn are excitatory in nature and are associated with strong or weak PKC $\gamma$ expression. Overall, Cre expression in Tac2-
Cre mice is precise and efficient in those neurons with persistent Tac2 expression, as demonstrated by double staining results (Figure $2 \mathrm{~B}$ ). However, the lineage tracing experiments also revealed a set of spinal neurons and peripheral sensory neurons that might express Tac2 transiently.

The expression pattern of Tomato in the dorsal horn of Tac2-Cre/Tomato mice is largely consistent with existing literature, but also differed in a few of respects 


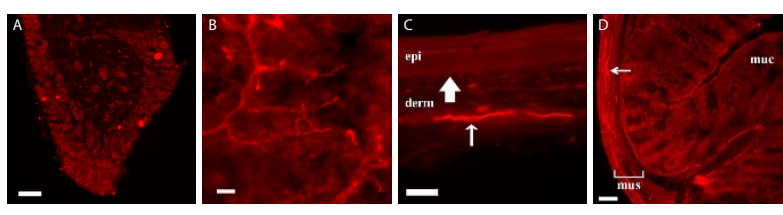

Figure 5 Tac2-Cre-expressing neurons in the DRG and their innervation of peripheral targets. Adult Tac2-Cre/ROSA26-

tdTomato mice showed fluorescence labeling in a small subset of lumbar DRG neurons (A). Their primary afferents innervated the glabrous skin in a wholemount preparation (B), and a transverse section showed selective innervation within the dermal layer (C, arrow). "derm": dermis and "epi": epidermis, and the solid arrow indicates boundary between dermis and epidermis. In a transverse section of the colon, Tomato-positive fibers were detected in the muscle layer (D, "mus"), by not in the mucousal layer (D, "muc").

from the NKB immunostaining pattern observed in the rat dorsal horn [16]. For example, whereas many NKBimmunostained neurons were observed in dorsal horn lamina I in rat [16], Tomato-positive neurons were only occasionally seen in lamina I of Tac2-Cre/Tomato mice (Figure 2B), consistent with in situ expression analyses $[14,27]$. This discrepancy could be due to species difference and/or the binding of the NKB antibody to certain NKB-negative neurons.

One notable feature of this Tac2-Cre mouse line, at least in the dorsal spinal cord, is the post-natal onset of Tac2-Cre expression (from P4-P10). Consequently, conditional knockouts in combination with this Cre line will be useful for studying the roles of interested genes in controlling postnatal development of neural circuits or controlling physiological functions of dorsal horn neurons.

Several recent genetic marking studies have led to identification of somatic sensory neurons that innervate specific peripheral targets. For example, MrgprD-expressing polymodal nociceptors, representing $30 \%$ of DRG neurons, innervate exclusively skin epidermis [34,35]. Similarly, MrgprB4-expressing neurons innervate skin epidermis around the hairs [36]. To our knowledge, Tac2-Cre/Tomato may represent the first molecular marker of a small subset of DRG neurons that innervate selective deep tissues, such as the dermis and visceral organs.

Behavioral studies suggested that the loss of Tac2 did not result in a detectable effect on pain or itch sensation, at least based on the assays used in this study. This finding is surprising considering that intrathecal injection of NKB or NK3R agonist has been reported to cause hyperalgesia [18,19] or hypoalgesia [17]. However, it is consistent with observations that no obvious pain deficits had been reported in NK3 knockout mice [37-39]. Perhaps the lack of behavioral deficit in Tac2 null mice is the result of redundant function with SP and NKA. Future studies on mice that lack both Tac1 and Tac 2 could be informative in this regard.

\section{Conclusions}

The Tac2-Cre knock-in mouse line is able to mark Tac2 lineage neurons in the dorsal root ganglia, the dorsal spinal cord and various parts of the brain. Thus, this Cre line will be a useful genetic tool for conditionally manipulating genes in selective populations of neurons.

\section{Methods}

\section{Generation of Tac2-Cre targeting Construct}

A $10 \mathrm{~Kb}$ EagI/XbaI fragment, containing all 8 exons of the Tac2 gene, was subcloned from the BAC RP23270B22 (UCSC Mus musculus Genome Brower). Next, the Cre coding sequence with a polyA signal was inserted in-frame with the first ATG of the Tac2 coding sequence. Finally, an FRT flanked neomycin cassette was inserted downstream of the Cre cassette. The orientation and sequence of the targeting construct was confirmed by sequencing.

\section{Generation of Tac2-Cre mice}

The targeting construct was linearized using NotI and electroporated into $129 / \mathrm{Sv}$ embryonic stem (ES) cells. ES cells were drug selected using geneticin and correctly targeted clones were identified using Southern blot analysis. Genomic DNA was extracted from 96 well colonies, digested with XbaI, Southern blotted, and hybridized using both 5' and 3' arm external probes. Correctly targeted Tac2 alleles exhibited two distinct 7 $\mathrm{kb}$ targeted bands, in contrast to the $12 \mathrm{~Kb}$ wildtype band. Correctly targeted clones were used by the DanaFarber ES cell core facility to generate transgenic mice via blastocyst injection. After establishing germ-line transmission, the neomycin cassette was excised by crossing to an FLP deleter strain [25] to generate the Tac2-Cre mouse line. Genotyping for the Tac2-Cre wildtype and mutant alleles was performed using PCR with Tac2 sense, Tac2 antisense, and Cre primers. Primer sequences were as follows: Tac2 sense primer $\left(5^{\prime}\right.$ - atccaacgctcttgagatcagggctcagat - 3'), Tac2 antisense primer (5'-ggacatcttaccttactgag-3'), Cre primer (5'-tcgaccggtaatgcaggcaa-3'). The product sizes were as follows: wildtype allele, Tac2 sense and antisense: 256 bp; mutant allele, Tac2 sense and Cre: 216 bp. Products were electrophoresed on $1.5 \%$ agarose gels, stained with ethidium bromide and photographed.

\section{Animals}

Heterozygous or homozygous Tac2-Cre mice were crossed to heterozygous or homozygous ROSA26-tdTomato mice (Madisen et al., 2010). Offspring were genotyped by PCR for Tac2-Cre and ROSA26-tdTomato 

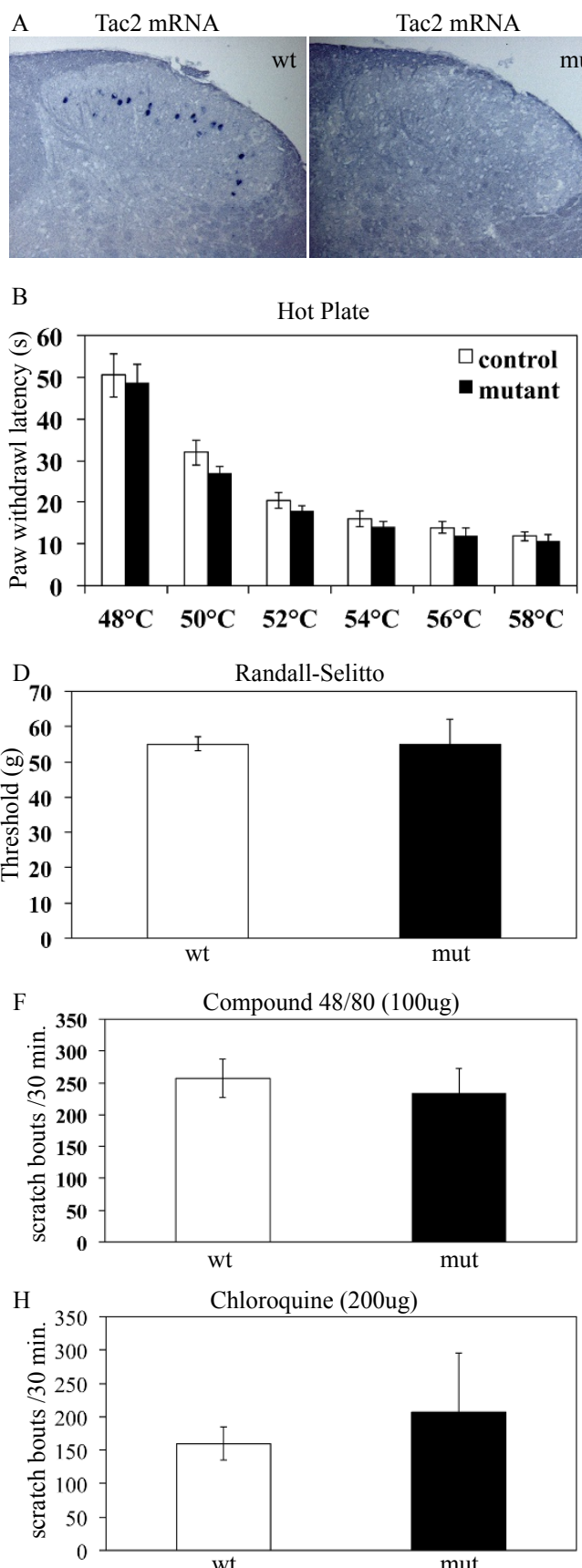

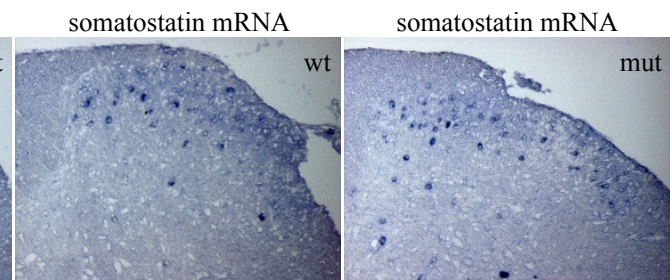

$\mathrm{C}$

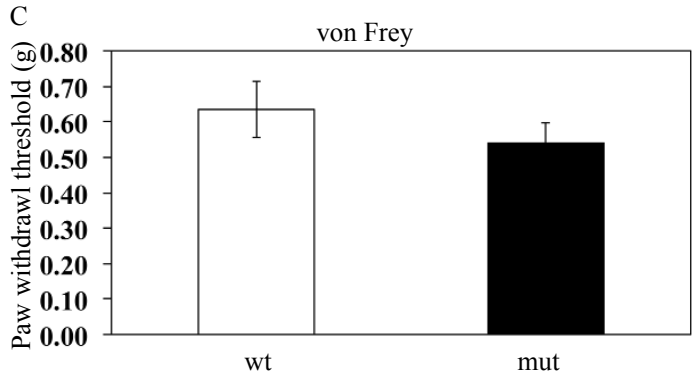

E

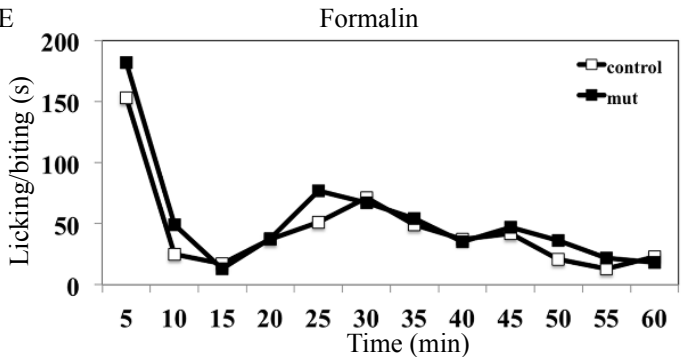

G PAR2 agonist (100ug)

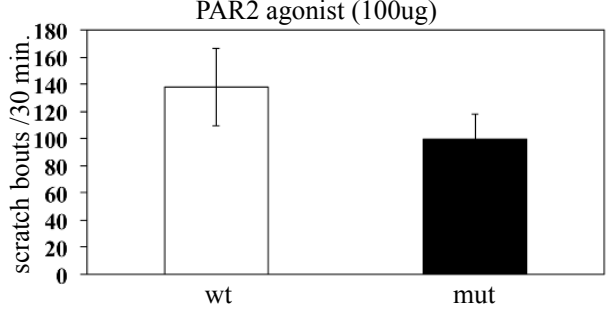

I

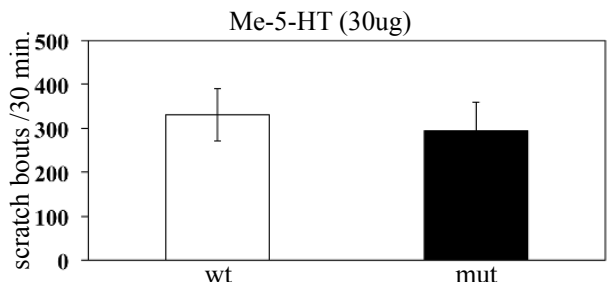

Figure 6 Tac2 Mutant mice did not exhibit defects in pain and itch sensation. A: In situ hybridization analyses of wildtype and mutant spinal cords using Tac2 and somatostatin antisense probes demonstrated the loss of Tac2 expression in mutant mice, while the expression of the somatostatin neuropeptide gene was unaffected. B-I: Responses to nociceptive and pruritic stimuli were normal in homozygous Tac2-Cre mice. Mutant (mut) Tac2-Cre mice were indistinguishable from wildtype (wt) littermates in terms of latency of hindpaw withdrawal upon application of various noxious temperatures (B, $p=0.19,0.33,0.09,0.71,0.39,0.40 ; 3$ female controls, 3 male controls, 4 female mutants, 3 male mutants); in mechanical threshold for eliciting hindpaw withdrawal following application of graded pressure by von Frey fibers $(\mathbf{C}, p=0.48 ; 12$ female controls, 7 male controls, 9 female mutants, 12 male mutants), and to the tail by the Randall-Selitto apparatus (D, $p=1.0 ; 3$ female controls, 2 female mutants, 2 male mutants). Licking and biting responses to hindpaw formalin injections were unaffected in the first phase, the first 10 minutes post-injection, and the second phase, 10-60 minutes post-injection ( $\mathbf{E}, p=0.32 ; 4$ female controls, 3 male controls, 5 female mutants, 2 male mutants). Furthermore, there was no significant difference in itch-related scratching responses following administration at the indicated dosages of pruritic agents including Compound $48 / 80(\mathbf{F}, p=0.65 ; 4$ female controls, 1 male control, 4 female mutants, 4 male mutants); PAR2 agonist $(\mathbf{G} n=8 ; p=0.29 ; 8$ female controls, 8 male controls, 8 female mutants, 8 male mutants); Chloroquine $(\mathbf{H}, p=0.64 ; 4$ female controls, 4 female mutants, 3 male mutants); and Me-5-HT (I, $p=0.33 ; 8$ female controls, 8 male controls, 8 female mutants, 8 male mutants). 
alleles. Double heterozygous Tac2-Cre and ROSA26tdTomato mice at P30 were used in immunohistochemical studies. Eight-week-old homozygous Tac2-Cre and control littermate mice were used in behavior analyses. The Institutional Animal Care and Use Committee at Dana-Farber Cancer Institute approved all experimental and behavioral test procedures.

\section{In situ hybridization (ISH) and Immunostaining}

ISH procedure of the Tac2 probe was previously described $[27,40]$. Briefly, mice were deeply anesthetized and perfused through the left cardiac ventricle with a fixative containing $4 \%$ freshly depolymerized paraformaldehyde (4\% PFA). Spinal cords were dissected and fixed overnight in $4 \%$ PFA, equilibrated overnight with $20 \%$ sucrose, and frozen in OCT (O.C.T. Compounds, BDH) on dry ice. $12 \mathrm{uM}$ sections were prepared and gently permeated by Proteinase $\mathrm{K}$, treated with triethanolamine, and hybridized with $1-2 \mu \mathrm{g} / \mathrm{mL}$ of DIG labeled antisense probe for over 12 hours. Non-specific binding was washed away by $0.2 \mathrm{xSSC}$ stringency at $60^{\circ} \mathrm{C}$, blocked by sheep serum and incubated with Alkaline phosphatase conjugated anti-DIG antibody. Alkaline phosphate reaction was catalyzed by NBT and BCIP (Roche). For tdTomato/ISH double staining, the Tomato fluorescent signal was directly photographed followed by performing ISH. The ISH signals were pseudocolored and then overlayed with the Tomato signal.

\section{Immunohistochemical Analyses}

Tissues were collected, fixed, and frozen as for ISH. 12 $\mu \mathrm{m}$ cryosections were dried at room temperature for 30 min and then fixed with 4\% PBS-buffered paraformaldehyde solution for $10 \mathrm{~min}$ on ice. After three washes in PBS, sections were incubated for $30 \mathrm{~min}$ in $10 \%$ goat serum diluted in PBS containing $0.3 \%$ Triton X-100 (PBST), and for $1 \mathrm{~h}$ at room temperature in 1:200 biotin conjugated IB4 $(10 \mathrm{mg} / \mathrm{ml}$, Sigma, USA) or 1:1000 dilution of a rabbit $\alpha$ PKC $\gamma$ antibody (SC-211, Santa Cruz), a 1:1000 dilution of a guinea pig $\alpha \mathrm{Tlx} 3$ antibody (a gift from C. Birchmeier, Germany), or a 1:1000 dilution of a rabbit $\alpha$-Pax 2 antibody (SC-211, Santa Cruz). Following three washes in PBST, the sections were incubated for 1 $\mathrm{h}$ in a 1:200 dilution of Alexa Fluor 488-Streptavidin (Invitrogen), Alexa Fluor 488-Goat- $\alpha$-Guinea Pig (Invitrogen), or Alexa Fluor 488-Goat $\alpha$-Rabbit (A11034, Invitrogen). After three washes in PBST, the sections were mounted in $90 \%$ glycerol, $0.5 \% \mathrm{~N}$-propyl gallate (pH 8.0) and analyzed using a fluorescent microscope.

\section{Behavioral measurements}

The experimenter was blind to which group an animal belonged.
Hot Plate test: naïve, awake, and unrestrained mice were individually removed from their home cage and placed on a metal surface pre-heated and maintained at the indicated temperatures (IITC Model PE34M-HC). The mice were enclosed within transparent $20 \mathrm{~cm}$ high walls, in a $14 \times 14 \mathrm{~cm}$ area Plexiglas chamber. The latency to performing a hindpaw flutter/shake, lick, or jump (whichever occurred first) was measured to the nearest $0.1 \mathrm{~s}$. The cut-off time for $48-52^{\circ} \mathrm{C}$ was $60 \mathrm{sec}-$ onds, while for $54-58^{\circ} \mathrm{C}$ it was 30 seconds.

von Frey threshold: awake, unrestrained, and acclimatized mice were individually removed from their home cage and habituated for $30 \mathrm{~min}$ in a metal mesh enclosure for 2 days. Mechanical sensitivity threshold of the stimulated hindpaw was measured for the next 2 consecutive days. Mechanical sensitivity was measured by determining the median $50 \%$ foot withdrawal threshold (measured in $\mathrm{mN}$ of discrete bending force), when a single prick to the hindfoot was applied steadily ( $>1 \mathrm{~s}$ duration) with a von Frey monofilament via the up-down method.

Randall-Selitto: A Randall-Selitto apparatus (Ugo Basile, Italy) was used to measure the withdrawal threshold. The animals were restrained in plastic enclosures and treated gently during the experiments. A cone-shaped pusher with a rounded tip (diameter of the base: $9 \mathrm{~mm}$ ) was applied to the tail. The intensity of pressure causing an escape reaction was defined as the withdrawal threshold. Measurements were performed 3 times at intervals of several minutes, and the mean value was taken as the threshold.

Formalin test: awake, unrestrained, and acclimatized mice were individually removed from their home cage and habituated for $30 \mathrm{~min}$ in mesh enclosures. Then, 25 $\mu \mathrm{L}$ of $5 \%$ formalin was injected subcutaneously into the plantar surface of the right hindpaw using a $0.5 \mathrm{~mL}$ insulin syringe with a $28 \mathrm{G} 1 / 2$ needle. Mice were then returned to the mesh enclosure, and behavioral observations were video taped. Video recording was subsequently played back, and the total time spent licking and/or biting the right hindpaw over the next $60 \mathrm{~min}$ utes was recorded to the nearest second in 5-min blocks. The early/acute phase was defined as 0-10 min post-injection, and the late/tonic phase as $10-60 \mathrm{~min}$ post-injection.

Itch assay: awake and unrestrained mice were individually removed from their home cage and acclimatized for 1 hour daily, for 5 continuous days, in Plexiglas containers $(10 \times 10 \times 12 \mathrm{~cm})$ atop a glass floor. At least two days before the injections, the nape of the neck (approximately $15 \times 10 \mathrm{~mm}$ area) was shaved after brief anesthesia with isoflurane (2\% in 100\% oxygen). During the experiment, a camcorder (Sony Model DCR-SR220) 
was positioned to video-record the scratching behavior of mice. $100 \mu \mathrm{g}$ of Compound 48/80 (Sigma-Aldrich), $100 \mu \mathrm{g}$ of PAR2 agonist SLIGRL- $\mathrm{NH}_{2}$ (Bachem), or 30 $\mu \mathrm{g}$ of serotonin agonist $\alpha$-Me-5-HT (Tocris) in $50 \mu \mathrm{l}$ of sterile saline was injected intradermally into the nape using a $0.5 \mathrm{ml}$ insulin syringe with a $28 \mathrm{G} 1 / 2$ needle (Becton-Dickinson). On the day of the experiment, mice were placed in containers and habituated for $15 \mathrm{~min}$. Then, the behavior of the mice was video recorded 30 min before (baseline behavior), and $30 \mathrm{~min}$ after, the chemical was injected. The experimenter was present briefly - once to start the video recording, and once later to administer the chemical. The video recording was subsequently played back and scratching bouts or wipes were counted. A scratching bout was initiated by lifting of the hind paw to the region to be scratched, and ended when the hind paw was returned to the floor or to the mouth for licking. Only the bouts or wipes to the shaved region were counted.

\section{Statistical Analyses of Pain and Itch Behaviors}

All data are presented as mean +/- SEM. Hot plate, von Frey, and Randall-Selitto data was calculated as the average of two independent tests performed on two consecutive days. For formalin and itching behaviors, the mean numbers of licking/biting and scratching bouts, respectively, and SEM during the period were calculated for each group. The difference between the mutant and control group was subjected to a Student's t test (twosample assuming unequal variance), with $\mathrm{p}<0.05$ considered statistically significant.

\section{Acknowledgements}

We thank Dr. James Horner for help in generating Tac2-Cre mice and Dr. Anthony Hill for help in acquiring confocal images.

\section{Authors' contributions}

QM conceived and supervised the conduct of the study. LM generated the Tac2-Cre mice and carried out all the molecular and behavioral analyses. LM and FCY carried out peripheral innervation analyses. LM and QM analyzed the molecular and behavioral data. LM and QM drafted the article, with help in editing from FCY. All authors read and approved the final manuscript.

\section{Competing interests}

The authors declare that they have no competing interests.

Received: 15 December 2011 Accepted: 24 January 2012

Published: 24 January 2012

\section{References}

1. Perl ER: Ideas about pain, a historical view. Nat Rev Neurosci 2007, 8:71-80.

2. Perl ER: Pain mechanisms: a commentary on concepts and issues. Prog Neurobiol 2011, 94:20-38

3. Todd AJ: Neuronal circuitry for pain processing in the dorsal horn. Nat Rev Neurosci 2010, 11:823-836.

4. Basbaum Al, Bautista DM, Scherrer G, Julius D: Cellular and molecular mechanisms of pain. Cell 2009, 139:267-284.

5. Mantyh PW, Rogers SD, Honore P, Allen BJ, Ghilardi JR, Li J, Daughters RS, Lappi DA, Wiley RG, Simone DA: Inhibition of hyperalgesia by ablation of lamina I spinal neurons expressing the substance P receptor. Science 1997, 278:275-279.

6. Sun YG, Zhao ZQ, Meng XL, Yin J, Liu XY, Chen ZF: Cellular basis of itch sensation. Science 2009, 325:1531-1534.

7. Carstens EE, Carstens MI, Simons CT, Jinks SL: Dorsal horn neurons expressing NK-1 receptors mediate scratching in rats. Neuroreport 2010, 21:303-308.

8. Neumann S, Braz JM, Skinner K, Llewellyn-Smith IJ, Basbaum Al: Innocuous, not noxious, input activates PKCgamma interneurons of the spinal dorsal horn via myelinated afferent fibers. J Neurosci 2008, 28:7936-7944.

9. Malmberg AB, Chen C, Tonegawa S, Basbaum Al: Preserved acute pain and reduced neuropathic pain in mice lacking PKCgamma. Science 1997, 278:279-283

10. Otsuka M, Yoshioka K: Neurotransmitter functions of mammalian tachykinins. Physiol Rev 1993, 73:229-308.

11. Cao YQ, Mantyh PW, Carlson EJ, Gillespie AM, Epstein CJ, Basbaum Al: Primary afferent tachykinins are required to experience moderate to intense pain. Nature 1998, 392:390-394.

12. Amatya B, El-Nour H, Holst M, Theodorsson E, Nordlind K: Expression of tachykinins and their receptors in plaque psoriasis with pruritus. $\mathrm{Br} J$ Dermatol 2011, 164:1023-1029.

13. Smith ES, Blass GR, Lewin GR, Park TJ: Absence of histamine-induced itch in the African naked mole-rat and "rescue" by Substance P. Mol Pain 2010, 6:29.

14. Lein ES, Hawrylycz MJ, Ao N, Ayres M, Bensinger A, Bernard A, Boe AF, Boguski MS, Brockway KS, Byrnes EJ, et al: Genome-wide atlas of gene expression in the adult mouse brain. Nature 2007, 445:168-176.

15. Warden MK, Young WS: Distribution of cells containing mRNAs encoding substance $\mathrm{P}$ and neurokinin $\mathrm{B}$ in the rat central nervous system. J Comp Neurol 1988, 272:90-113.

16. Polgar E, Furuta T, Kaneko T, Todd A: Characterization of neurons that express preprotachykinin $B$ in the dorsal horn of the rat spinal cord. Neuroscience 2006, 139:687-697.

17. Laneuville O, Dorais J, Couture R: Characterization of the effects produced by neurokinins and three agonists selective for neurokinin receptor subtypes in a spinal nociceptive reflex of the rat. Life Sci 1988, 42:1295-1305.

18. Linden DR, Seybold VS: Spinal neurokinin3 receptors mediate thermal but not mechanical hyperalgesia via nitric oxide. Pain 1999, 80:309-317.

19. Linden DR, Jia YP, Seybold VS: Spinal neurokin3 receptors facilitate the nociceptive flexor reflex via a pathway involving nitric oxide. Pain 1999, 80:301-308.

20. Zhao J, Nassar MA, Gavazzi I, Wood JN: Tamoxifen-inducible NaV1.8CreERT2 recombinase activity in nociceptive neurons of dorsal root ganglia. Genesis 2006, 44:364-371.

21. Agarwal N, Offermanns S, Kuner R: Conditional gene deletion in primary nociceptive neurons of trigeminal ganglia and dorsal root ganglia. Genesis 2004, 38:122-129.

22. Abrahamsen B, Zhao J, Asante CO, Cendan CM, Marsh S, MartinezBarbera JP, Nassar MA, Dickenson AH, Wood JN: The cell and molecular basis of mechanical, cold, and inflammatory pain. Science 2008, 321:702-705.

23. Liu Y, Abdel Samad O, Zhang L, Duan B, Tong Q, Lopes C, Ji RR, Lowell BB, Ma Q: VGLUT2-dependent glutamate release from nociceptors is required to sense pain and suppress itch. Neuron 2010, 68:543-556.

24. Skarnes WC, Rosen B, West AP, Koutsourakis M, Bushell W, Iyer V, Mujica AO, Thomas M, Harrow J, Cox T, et al: A conditional knockout resource for the genome-wide study of mouse gene function. Nature 2011, 474:337-342.

25. Farley FW, Soriano P, Steffen LS, Dymecki SM: Widespread recombinase expression using FLPeR (flipper) mice. Genesis 2000, 28:106-110.

26. Madisen L, Zwingman TA, Sunkin SM, Oh SW, Zariwala HA, Gu H, Ng LL, Palmiter RD, Hawrylycz MJ, Jones AR, et al: A robust and high-throughput Cre reporting and characterization system for the whole mouse brain. Nat Neurosci 2010, 13:133-140.

27. Xu Y, Lopes C, Qian Y, Liu Y, Cheng L, Goulding M, Turner EE, Lima D, Ma Q: TIx1 and TIx3 coordinate specification of dorsal horn painmodulatory peptidergic neurons. J Neurosci 2008, 28:4037-4046.

28. Cheng L, Arata A, Mizuguchi R, Qian Y, Karunaratne A, Gray PA, Arata S, Shirasawa S, Bouchard M, Luo P, et al: Tlx3 and Tlx1 are post-mitotic selector genes determining glutamatergic over GABAergic cell fates. Nat Neurosci 2004, 7:510-517. 
29. Shepherd GM, Chen WR, Willhite D, Migliore M, Greer CA: The olfactory granule cell: from classical enigma to central role in olfactory processing. Brain research reviews 2007, 55:373-382.

30. Westbrook GL: Seizures and Epilepsy. In Principles of Neural Science. Edited by: Kandel ER, Schwartz, James H, Jessell, Thomas M. New York: McGrawHill; 2000:910-935.

31. Park TJ, Lu Y, Juttner R, Smith ES, Hu J, Brand A, Wetzel C, Milenkovic N, Erdmann B, Heppenstall PA, et al: Selective inflammatory pain insensitivity in the African naked mole-rat (Heterocephalus glaber). PLoS biology 2008, 6:e13.

32. Shimada SG, LaMotte RH: Behavioral differentiation between itch and pain in mouse. Pain 2008, 139:681-687.

33. Shimada SG, Shimada KA, Collins JG: Scratching behavior in mice induced by the proteinase-activated receptor-2 agonist, SLIGRL-NH2. Eur J Pharmacol 2006, 530:281-283.

34. Zylka MJ, Rice FL, Anderson DJ: Topographically distinct epidermal nociceptive circuits revealed by axonal tracers targeted to Mrgprd. Neuron 2005, 45:17-25.

35. Jimenez-Andrade JM, Mantyh WG, Bloom AP, Xu H, Ferng AS, Dussor G, Vanderah TW, Mantyh PW: A phenotypically restricted set of primary afferent nerve fibers innervate the bone versus skin: therapeutic opportunity for treating skeletal pain. Bone 2010, 46:306-313.

36. Liu Q, Vrontou S, Rice FL, Zylka MJ, Dong X, Anderson DJ: Molecular genetic visualization of a rare subset of unmyelinated sensory neurons that may detect gentle touch. Nat Neurosci 2007, 10:946-948.

37. Kung TT, Crawley Y, Jones H, Luo B, Gilchrest H, Greenfeder S, Anthes JC, Lira S, Wiekowski M, Cook DN, et al: Tachykinin NK3-receptor deficiency does not inhibit pulmonary eosinophilia in allergic mice. Pharmacol Res 2004, 50:611-615.

38. Siuciak JA, McCarthy SA, Martin AN, Chapin DS, Stock J, Nadeau DM Kantesaria S, Bryce-Pritt D, McLean S: Disruption of the neurokinin-3 receptor (NK3) in mice leads to cognitive deficits. Psychopharmacology (Berl) 2007, 194:185-195.

39. Nordquist RE, Savignac H, Pauly-Evers M, Walker G, Knoflach F, Borroni E, Glaentzlin P, Bohrmann B, Messer J, Ozmen L, et al: Characterization of behavioral response to amphetamine, tyrosine hydroxylase levels, and dopamine receptor levels in neurokinin 3 receptor knockout mice. Behav Pharmacol 2008, 19:518-529.

40. Gray PA, Fu H, Luo P, Zhao Q, Yu J, Ferrari A, Tenzen T, Yuk DI, Tsung EF, Cai $Z$, et al: Mouse brain organization revealed through direct genomescale TF expression analysis. Science 2004, 306:2255-2257.

doi:10.1186/1756-6606-5-3

Cite this article as: Mar et al.: Genetic marking and characterization of Tac2-expressing neurons in the central and peripheral nervous system. Molecular Brain 2012 5:3.

\section{Submit your next manuscript to BioMed Central and take full advantage of:}

- Convenient online submission

- Thorough peer review

- No space constraints or color figure charges

- Immediate publication on acceptance

- Inclusion in PubMed, CAS, Scopus and Google Scholar

- Research which is freely available for redistribution 